\title{
CoERG11 A395T mutation confers azole resistance in Candida orthopsilosis clinical isolates
}

\author{
Cosmeri Rizzato ${ }^{1}$, Noemi Poma ${ }^{2}$, Marina Zoppo ${ }^{2}$, Brunella Posteraro ${ }^{3}$, Enrica Mello ${ }^{4}$, Daria Bottai ${ }^{2}$, \\ Antonella Lupetti ${ }^{1}$, Maurizio Sanguinetti ${ }^{4}$ and Arianna Tavanti ${ }^{2 *}$
}

${ }^{1}$ Department of Translational Research and New Technologies in Medicine and Surgery, University of Pisa, Pisa, Italy; ${ }^{2}$ Department of Biology, University of Pisa, Pisa, Italy; ${ }^{3}$ Institute of Public Health, Università Cattolica del Sacro Cuore, Fondazione Policlinico Universitario Agostino Gemelli, Rome, Italy; ${ }^{4}$ Institute of Microbiology, Università Cattolica del Sacro Cuore, Fondazione Policlinico Universitario Agostino Gemelli, Rome, Italy

*Corresponding author. Tel: +39-050-2213965; Fax: +39-050-2213711; E-mail: arianna.tavanti@unipi.it †These authors contributed equally to this work.

Received 7 December 2017; returned 7 February 2018; revised 5 March 2018; accepted 7 March 2018

Background: Candida orthopsilosis is a human fungal pathogen responsible for a wide spectrum of symptomatic infections. Evidence suggests that C. orthopsilosis is mainly susceptible to azoles, the most extensively used antifungals for treatment of these infections. However, fluconazole-resistant clinical isolates are reported.

Objectives: This study evaluated the contribution of a single amino acid substitution in the azole target CoErg11 to the development of azole resistance in C. orthopsilosis.

\begin{abstract}
Methods: C. orthopsilosis clinical isolates $(n=40)$ were tested for their susceptibility to azoles and their CoERG11 genes were sequenced. We used a SAT1 flipper-driven transformation to integrate a mutated CoERG11 allele in the genetic background of a fluconazole-susceptible isolate.
\end{abstract}

Results: Susceptibility testing revealed that 16 of $40 \mathrm{C}$. orthopsilosis clinical isolates were resistant to fluconazole and to at least one other azole. We identified an A395T mutation in the CoERG11 coding sequence of azoleresistant isolates only that resulted in the non-synonymous amino acid substitution Y132F. The SAT1 flipper cassette strategy led to the creation of $C$. orthopsilosis mutants that carried the A395T mutation in one or both CoERG11 alleles (heterozygous or homozygous mutant, respectively) in an azole-susceptible genetic background. We tested mutant strains for azole susceptibility and for hot-spot locus heterozygosity. Both the heterozygous and the homozygous mutant strains exhibited an azole-resistant phenotype.

Conclusions: To the best of our knowledge, these findings provide the first evidence that the CoErg11 Y132F substitution confers multi-azole resistance in C. orthopsilosis.

\section{Introduction}

Severe fungal infections afflict millions of patients, resulting in $>1350000$ deaths annually, ${ }^{1}$ often as a consequence of failure to rapidly treat patients because of delayed or missed diagnosis. ${ }^{2}$ Candida orthopsilosis is a human fungal pathogen belonging to the Candida parapsilosis species complex. ${ }^{3}$ These species colonize virtually all body sites and cause a wide spectrum of symptomatic human infections, ranging from mucosal to life-threatening invasive diseases. ${ }^{4}$ While Candida albicans remains the most frequently isolated Candida species, ${ }^{5}$ studies conducted in Italy, Spain and Latin America show that the C. parapsilosis species complex ranks as the second most common cause of bloodstream infection. ${ }^{6-8}$ Although less prevalent than C. parapsilosis, the two sibling C. orthopsilosis and Candida metapsilosis species are often isolated from the blood of paediatric patients, including neonates. ${ }^{8-12}$ In this population in particular, the antifungal agent fluconazole is widely used as a prophylactic agent to prevent candidaemia and other forms of invasive candidiasis. ${ }^{10}$

Among the azole resistance mechanisms known to date, genetic modifications of the ERG11-encoded lanosterol $14 \alpha$-demethylase target, resulting in a reduced affinity for the drug, influence the spectrum of azole activities. ${ }^{11}$ Fluconazole has the weakest interaction with the Erg11 target and displays the narrowest antifungal spectrum; it is therefore active against yeasts but not against other fungi. As such, fluconazole promotes the broadest antifungal resistance. ${ }^{11}$ Of the 70 Erg11 amino acid substitutions described in azole-resistant clinical isolates of $C$. albicans, some result in resistance only to fluconazole, others to voriconazole but not to posaconazole, and some others to all the azole class 
members. $^{12-17}$ Computational modelling using high-resolution structures as a template may help to explain the impact of specific amino acid substitutions on drug-target interactions in Candida species. ${ }^{18,19}$ However, due to very low incidence (usually $<5 \%$ ) of C. orthopsilosis candidaemia, ${ }^{20,21}$ only a small number of studies addressing fluconazole resistance mechanisms are available for this species. $^{22-26}$

In this study, we sought to investigate the molecular mechanisms of azole resistance in C. orthopsilosis by using a set of selected azole-susceptible and azole-resistant clinical isolates. To the best of our knowledge, this is the first study demonstrating the role of the A395T CoERG11 (C. orthopsilosis ERG11) mutation in the azole resistance of $C$. orthopsilosis.

\section{Materials and methods}

\section{Strains and growth conditions}

The C. orthopsilosis clinical isolates used in this study were collected at the Microbiology Unit of Pisa University Hospital (Pisa, Italy) and at the Institute of Microbiology of the Università Cattolica del Sacro Cuore, Fondazione Policlinico Universitario Agostino Gemelli (Rome, Italy) (see Table 1). Isolates were obtained from single patients and were identified by MALDITOF MS (Bruker Daltonics, Bremen, Germany). Isolates were stored at $-80^{\circ} \mathrm{C}$ in yeast/peptone/dextrose (YPD) broth supplemented with $40 \%$ glycerol, and subcultured on YPD agar plates at $30^{\circ} \mathrm{C}$ when necessary. Prior to use, isolates were grown in $\mathrm{YPD}$ broth at $30^{\circ} \mathrm{C}$ under agitation. For selection of nourseothricin-resistant $C$. orthopsilosis recombinant strains, nourseothricin (Werner BioAgents, Jena, Germany) was added to YPD medium at a $100 \mathrm{mg} / \mathrm{L}$ final concentration. For excision of SAT1 flipper cassette, mutant strains were grown for $48 \mathrm{~h}$ at $30^{\circ} \mathrm{C}$ in $1 \times$ yeast nitrogen base (YNB) medium supplemented with $20 \mathrm{~g} / \mathrm{L}$ maltose. Escherichia coli DH5 $\alpha$ genotype $F-$ , endA1, hsdR17 [rk-, mk-], supE44, thi-1, recA1, gyrA96, relA1, $\Delta$ [argFlac]U169, $\lambda$-, Ф80dlacZ $\Delta M 15$ was used for plasmid construction and propagation. Bacterial cells were grown at $37^{\circ} \mathrm{C}$ in LB broth or on LB agar plates; when required, ampicillin (Sigma-Aldrich, St Louis, MO, USA) was added to a final concentration of $100 \mathrm{mg} / \mathrm{L}$. All mutant strains were kept in $40 \%$ glycerol stock frozen at $-80^{\circ} \mathrm{C}$.

\section{Antifungal susceptibility testing}

C. orthopsilosis clinical isolates and COERG11 mutant strains were tested for antifungal susceptibility to fluconazole, posaconazole, itraconazole and voriconazole, in accordance with EUCAST guidelines. ${ }^{27}$ MIC values were interpreted using species (C. parapsilosis)-specific EUCAST clinical breakpoints (http://www.eucast.org/clinicalbreakpoints/).

\section{CORT_OE05900 sequencing and in silico analysis}

Genomic DNA was isolated from C. orthopsilosis clinical isolates grown overnight in $10 \mathrm{~mL}$ of YPD medium at $30^{\circ} \mathrm{C}$ as previously described. ${ }^{28}$ The C. orthopsilosis ERG11 gene was identified by searching the http://cgob3. ucd.ie/database, which encompasses the annotated genomes of several clinically relevant Candida species, including C. orthopsilosis (strain 90-125). ${ }^{29}$ Sequencing of the CORT_OEO5900 gene, an orthologue of C. albicans ERG11 (http://cgob3.ucd.ie/), referred to above as CoERG11, was performed using overlapping primers in chromosomal order CORTOE05900f1-CORTOE05900r1 (574 bp, from nucleotide + 217 after the STOP codon to +1212), CORTOE05900f2-CORTOE05900r2 (871 bp, from nucleotide +1267 to +396 ) and CORTOE05900f3-CORTOE05900r3 ( $984 \mathrm{bp}$, from nucleotide +588 to -396 before the ATG codon). Primer sequences and parameters used for PCR amplification of specific gene fragments are shown in Table S1 and Figure S1 (available as Supplementary data at JAC Online). Gene sequences were deposited in GenBank under accession numbers: MG544857, MG495386, MG495387, MG495388, MG495389, MG495390, MG495385, MG516582, MG544858, MG516583, MG516584, MG543287, MG543288, MG543289, MG601484, MG601487, MG601488, MG601489, MG601490, MG584838, MG584839, MG584840, MG584841, MG584842, MG584843, MG584844, MG601485 and MG601486 (Table S2).

The effect of COERG11 DNA polymorphisms on the predicted protein was evaluated with three bioinformatics tools: SIFT (Sorting Intolerant From Tolerant; http://sift.jcvi.org), ${ }^{30}$ PHYRE2 (Protein Homology/analogY Recognition Engine v. 2.0; http://www.sbg.bio.ic.ac.uk/phyre2/html) ${ }^{31}$ and PROVEAN (PROtein Variation Effect ANalyser). ${ }^{32}$

\section{Construction of the SAT1 flipper cassette carrying the A395T CoERG11 mutation}

To transfer an entire functional copy of the CoERG11 gene bearing the A395T substitution, the plasmid PSFS2 containing the SAT1 flipper cassette elements was used as a genetic background to construct the transformation cassette. $^{33}$

A downstream 3' homology CoERG11 region (574 bp) was amplified by PCR using DreamTaq Polymerase (Thermo Scientific, Waltham, MA, USA) from the genome of C. orthopsilosis ATCC 96139 reference strain, using primers ERG11IFSacII and ERG11ERSacI containing engineered SacII and SacI restriction sites (Table S1). The PCR product was purified using the QIAquick PCR purification Kit (Qiagen, Milan, Italy) according to the manufacturer's guidelines. Next, PSFS2 plasmid DNA and the purified PCR product were both digested with SacI and SacII (New England Biolabs, Ipswich, MA, USA) and ligated using the T4 ligase enzyme (New England Biolabs), generating the P3'HOM.ERG11 plasmid (Table S3), which was propagated in E. coli $\mathrm{DH} 5 \alpha$-competent cells. An upstream 5' homology fragment (1716 bp) comprising the entire COERG11 coding sequence was amplified from a C. orthopsilosis fluconazole-resistant clinical isolate (Co681, MIC $=64 \mathrm{mg} / \mathrm{L}$ ) by PCR using Q5 Taq Polymerase (New England Biolabs) with primers ERG11EFApaI and ERG11ERXhoI containing ApaI- and XhoI-engineered sites (Table S1). Both COERG11 and P3'HOM.ERG11 plasmid were digested with ApaI and XhoI enzymes and ligated to generate the plasmid PERG11 (Table S3). Plasmid DNA was then extracted from E. coli and amplified using CORTOE05900Rtfwd and CORTOE05900r1 primers (Table S1). The PCR product was purified (Qiagen) for sequencing in order to verify the presence of the A395T mutation (GATC Biotech AG). The PERG11 plasmid was double digested with SacI and ApaI, producing a $6450 \mathrm{bp}$ fragment containing the SAT1 flipper cassette flanked by the COERG11 sequence at the $5^{\prime}$ end and the $3^{\prime}$ homology region. The cassette was gel purified and used in C. orthopsilosis transformation experiments.

\section{Transformation of a C. orthopsilosis fluconazole- susceptible strain with the SAT1 flipper construct carry- ing the A395T CoERG11 mutation}

The fluconazole-susceptible $C$. orthopsilosis Co287 (MIC = 2 mg/L) was selected as the parental strain for transformation experiments. C. orthopsilosis Co287 strain was transformed by electroporation as previously described ${ }^{34}$ with minor modifications. Approximately $4 \mu \mathrm{g}$ of gel-purified disruption cassette was electroporated in strain C0287 with the following conditions: $2.5 \mathrm{kV}, 25 \mu \mathrm{F}$ and 200 ohms (GenePulser, Bio-Rad, Milan, Italy). Negative and positive transformation controls were also included, to check for nourseothricin susceptibility and cell viability, respectively. Following a $3 \mathrm{~h}$ recovery in $100 \mu \mathrm{L}$ of fresh YPD medium at $30^{\circ} \mathrm{C}, 900 \mu \mathrm{L}$ of YPD was added and cells were plated on YPD agar supplemented with the selective agent (100 mg/L nourseothricin). Plates were incubated for $48 \mathrm{~h}$ at $30^{\circ} \mathrm{C}$. Nourseothricin-resistant clones were screened by PCR. Genomic DNA extraction was performed as previously described. ${ }^{35}$ Briefly, one colony was suspended in $100 \mu \mathrm{L}$ of a solution containing (200 mM LiOAc; $1 \%$ SDS) incubated at $70^{\circ} \mathrm{C}$ for $15 \mathrm{~min}$ and then precipitated with $96 \%$ ethanol. Correct 
Table 1. Antifungal susceptibility testing of C. orthopsilosis clinical isolates

\begin{tabular}{|c|c|c|c|c|c|c|c|c|c|c|}
\hline \multirow[b]{2}{*}{ Isolate } & \multicolumn{4}{|c|}{ MIC EUCAST (mg/L) } & \multicolumn{6}{|c|}{ Amino acid substitution } \\
\hline & POS & VRC & ITC & FLC & V58I & Y132F & Q211K & S420F & A421V & V485I \\
\hline Rome1 & 0.03 & 0.03 & 0.03 & 2 & V & Y & Q & $\mathrm{F}$ & A & V \\
\hline Rome2 & 0.03 & 0.03 & 0.03 & 1 & V & Y & Q & $\mathrm{F}$ & A & V \\
\hline Rome3 & 0.03 & 0.03 & 0.06 & 1 & V & Y & Q & $\mathrm{F}$ & A & V \\
\hline Rome4 & 0.06 & 0.06 & 0.25 & 2 & I & Y & Q & $S$ & A & V \\
\hline Rome5 & 0.06 & 0.06 & 0.125 & 2 & I & Y & Q & $S$ & A & V \\
\hline Rome6 & 0.06 & 0.125 & 0.125 & 4 & V & Y & Q & $\mathrm{F}$ & $A$ & V \\
\hline Rome7 & 0.25 & 0.25 & 0.25 & 2 & I & Y & Q & S & heterozygous & heterozygous \\
\hline Rome8 & 0.03 & 0.125 & 0.125 & 2 & I & Y & Q & $S$ & $A$ & V \\
\hline Rome9 & 0.125 & 0.25 & 0.25 & 4 & V & Y & K & $S$ & V & I \\
\hline Rome10 & 0.25 & 0.25 & 0.25 & 4 & V & Y & K & $S$ & V & I \\
\hline Rome11 & 0.25 & 0.125 & 0.25 & 4 & V & $Y$ & K & S & V & I \\
\hline Rome12 & 0.125 & 0.25 & 0.25 & 4 & V & Y & Q & $S$ & V & I \\
\hline Rome13 & 0.25 & 0.25 & 0.125 & 4 & V & Y & K & $S$ & V & I \\
\hline Rome14 & 0.25 & 0.06 & 0.125 & 4 & V & Y & K & $S$ & V & I \\
\hline Rome15 & 0.125 & 0.25 & 0.125 & 4 & V & Y & K & $S$ & V & I \\
\hline Rome17 & 0.125 & 0.125 & 0.25 & 4 & V & Y & K & $S$ & V & I \\
\hline Rome19 & 0.25 & 0.5 & 0.25 & 16 & V & $Y$ & K & $S$ & V & I \\
\hline Rome18 & 0.25 & 0.5 & 0.25 & 32 & V & Y & $Q$ & $S$ & V & I \\
\hline Rome22 & 0.5 & 4 & 0.5 & 128 & V & $\mathrm{F}$ & $Q$ & $S$ & V & I \\
\hline Rome23 & 0.125 & 2 & 0.25 & 64 & V & $\mathrm{F}$ & heterozygous & $S$ & V & I \\
\hline Rome24 & 0.125 & 4 & 0.125 & 128 & V & $\mathrm{F}$ & K & $S$ & V & I \\
\hline Rome25 & 0.25 & 2 & 0.25 & 128 & V & $\mathrm{F}$ & K & S & V & I \\
\hline Rome26 & 0.125 & 4 & 0.5 & 128 & V & $\mathrm{F}$ & heterozygous & $S$ & V & I \\
\hline Rome27 & 0.125 & 4 & 0.25 & 64 & V & $\mathrm{F}$ & K & S & V & I \\
\hline Rome28 & 0.25 & 8 & 0.25 & 64 & V & $\mathrm{F}$ & $Q$ & $S$ & V & I \\
\hline Rome29 & 0.125 & 4 & 0.25 & 64 & V & Y & Q & $S$ & V & I \\
\hline Co25 & 0.125 & 0.06 & 0.25 & 8 & V & Y & K & $S$ & V & I \\
\hline Co47 & 0.125 & 0.25 & 0.25 & 2 & V & Y & K & $S$ & V & I \\
\hline Co85 & 0.03 & 0.125 & 0.25 & 4 & V & $Y$ & Q & $S$ & A & V \\
\hline Co124 & 0.06 & 0.06 & 0.5 & 8 & V & $Y$ & $Q$ & $\mathrm{~F}$ & A & V \\
\hline Co242 & 0.125 & 0.06 & 0.25 & 8 & V & $Y$ & $Q$ & $S$ & A & V \\
\hline Co268 & 0.125 & 0.06 & 0.25 & 16 & V & $Y$ & $Q$ & $\mathrm{~F}$ & A & V \\
\hline Co269 & 0.125 & 0.125 & 0.125 & 4 & V & Y & $Q$ & $\mathrm{~F}$ & A & V \\
\hline Co287 & 0.03 & 0.125 & 0.125 & 2 & V & $Y$ & $\mathrm{Q}$ & $S$ & A & V \\
\hline Co289 & 0.125 & 0.125 & 0.5 & 4 & V & $\mathrm{F}$ & $Q$ & $S$ & V & I \\
\hline Co296 & 0.125 & 0.5 & 0.5 & 4 & V & $Y$ & $Q$ & $\mathrm{~F}$ & A & V \\
\hline Co331 & 0.06 & 0.5 & 0.25 & 4 & V & $Y$ & K & $S$ & V & I \\
\hline Co681 & 0.125 & 2 & 0.5 & 64 & $\mathrm{~V}$ & $\mathrm{~F}$ & K & $S$ & $\mathrm{~V}$ & I \\
\hline Co687 & 0.25 & 2 & 0.25 & 64 & V & $\mathrm{F}$ & K & $S$ & V & I \\
\hline Co690 & 0.03 & 0.5 & 0.25 & 1 & V & $Y$ & K & $S$ & V & I \\
\hline
\end{tabular}

POS, posaconazole (R, >0.06 mg/L); VRC, voriconazole (R, >0.25 mg/L); ITC, itraconazole ( $R,>0.125 \mathrm{mg} / \mathrm{L})$; FLC, fluconazole $(\mathrm{R},>4 \mathrm{mg} / \mathrm{L})$.

integration of the cassette was verified using primers Sat1F and OutERG11R (Table S1). Clones that had correctly integrated the cassette were then grown on YNB supplemented with maltose to allow excision of the cassette from the fungal genome. Correct excision was verified by PCR using primers CORTOE05900f1 and CORTOE05900f3 (Table S1). Heterozygosity of the obtained clones was verified by PCR using the primers ERG113HOMF and OutERG11R (Table S1). The presence of the A395T mutation was verified in clones NP1-4 by sequencing an internal fragment of CoERG11 gene, using PCR and sequencing primers CORTOE05900Rtfwd and CORTOE05900r1 (Table S1).

\section{Phenotypic assays on C. orthopsilosis mutants}

C. orthopsilosis Co287-derived mutants and the parental Co287 WT strain were tested for their growth ability in basal conditions. For each clone, a single colony was grown overnight at $37^{\circ} \mathrm{C}$ in $10 \mathrm{~mL}$ of YPD broth, all the strains were diluted to the same OD and were then subcultured in $50 \mathrm{~mL}$ of YPD at $37^{\circ} \mathrm{C}$ under agitation for $24 \mathrm{~h}$. Spectrophotometric readings were taken every $2 \mathrm{~h}$ for the first $6 \mathrm{~h}$ and then every hour up to $24 \mathrm{~h}$. Antifungal susceptibility testing of the mutant strains was performed as described above. 


\section{Results}

\section{C. orthopsilosis azole susceptibility testing and identification of CoErg11 amino acid variants}

Analysis of susceptibility data obtained from the 40 C. orthopsilosis clinical isolates included in the study indicated that 16 isolates were resistant to fluconazole and to at least one other azole antifungal (Table 1). We investigated the sequence variation of the CoERG11 gene in all fluconazole-resistant and fluconazole-susceptible C. orthopsilosis isolates. By comparison of the obtained sequences with that of the reference $C$. orthopsilosis isolate (90-125), ${ }^{29} 42$ different SNPs (Table S4) in 40 isolates were identified, six of which resulted in non-synonymous amino acid substitutions in the corresponding protein (Table 1). Among these, the A395T (amino acid substitution Y132F) was present in 10 of the azole-resistant isolates but in none of the susceptible ones. The other five SNPs were T173G (amino acid substitution V58I), C631A (amino acid substitution Q211K), C1259T (amino acid substitution S420F), C1262T (amino acid substitution A421V) and G1450A (amino acid substitution V485I). Notably, these mutations were present in both susceptible and resistant isolates (Table 1).

(a)

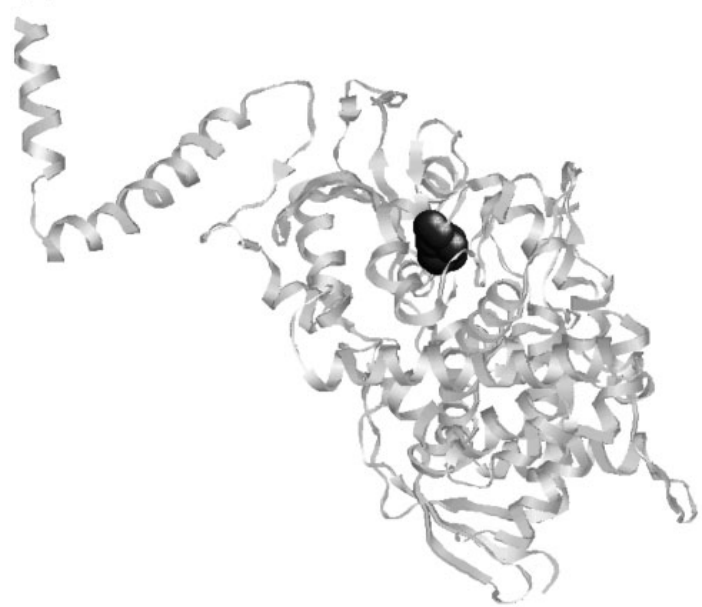

\section{In silico analysis of CoErg11 variants}

The impact of the six non-synonymous mutations identified in this study on C. orthopsilosis Erg11 protein function was predicted using the SIFT, PROVEAN and Phyre algorithms. The SIFT and PROVEAN algorithms predicted the mutation Y132F to be deleterious, potentially affecting protein functionality (score 0.02 and -3.761 , respectively) and although analysis with the Phyre suite found a low conservation of the amino acid in position 132, it also predicted a medium level of mutation sensitivity. This program also mapped the substituted amino acid into the active site of the protein (Figure 1). Mutations V58I, Q211K, S420F, A421V and V485I were predicted by the SIFT algorithm to be tolerated; none of them mapped into the protein pocket (data not shown).

\section{Construction of a C. orthopsilosis strain carrying a CoERG11 395 T mutated allele}

To assess the effect of the A395T mutation in the development of azole resistance in C. orthopsilosis, the entire CoERG11 coding sequence from an azole-resistant clinical isolate carrying the mutation was introduced into the $C$. orthopsilosis azole-susceptible isolate Co287, which is WT for the CoERG11 allele (Table 2), using

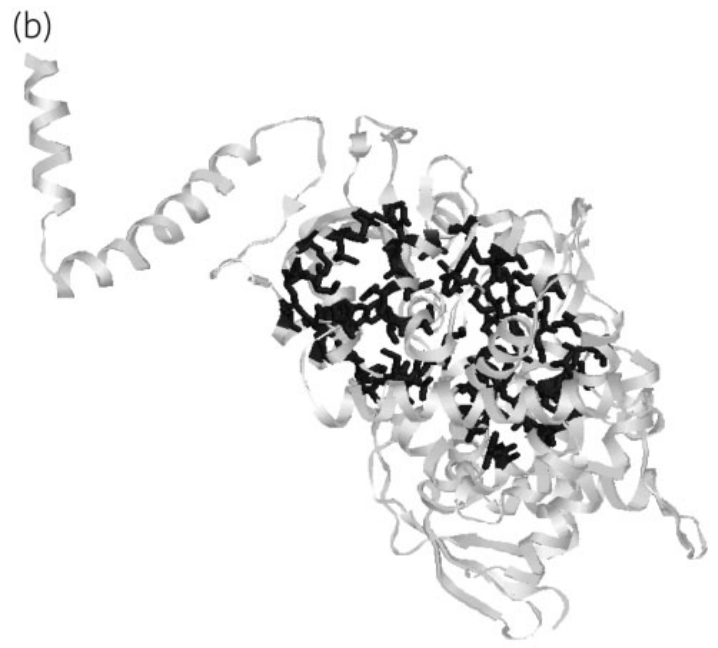

Figure 1. Prediction of PHYRE2 algorithm on the amino acid 132 position in C. orthopsilosis Erg11 protein (a) and visualization of the protein pocket (b) according to the C. albicans Erg11 resolved structure. ${ }^{19}$

Table 2. Genotyping and azole susceptibility testing of the C. orthopsilosis mutant clones obtained in this study

\begin{tabular}{|c|c|c|c|c|c|c|}
\hline \multirow[b]{2}{*}{ Strain } & \multirow[b]{2}{*}{ CoERG11 genotype } & \multirow[b]{2}{*}{ Amino acid 132 (CoErg11) } & \multicolumn{4}{|c|}{ MIC EUCAST (mg/L) } \\
\hline & & & POS & VRC & ITC & FLC \\
\hline Co287 & $E R G 11^{395 A} / E R G 11^{395 A}$ & $Y / Y$ & 0.03 & 0.03 & 0.25 & 1 \\
\hline NP1 & ERG11 ${ }^{395 T}: .: F R T / E R G 11^{395 A}$ & $\mathrm{Y} / \mathrm{F}$ & 0.06 & 1 & 1 & 16 \\
\hline NP2 & ERG11 ${ }^{395 T}: . F R T / E R G 11^{395 T}:$. FRT & $\mathrm{F} / \mathrm{F}$ & 0.06 & 1 & 1 & 32 \\
\hline NP3 & ERG11 $1^{395 T}:: F R T / E R G 11^{395 A}$ & $\mathrm{Y} / \mathrm{F}$ & 0.06 & 0.25 & 0.5 & 16 \\
\hline NP4 & ERG11395A.::FRT/ERG11 395A.::FRT & $\mathrm{Y} / \mathrm{Y}$ & 0.06 & 0.06 & 0.125 & 1 \\
\hline
\end{tabular}

POS, posaconazole; VRC, voriconazole; ITC, itraconazole; FLC, fluconazole. 
(a)

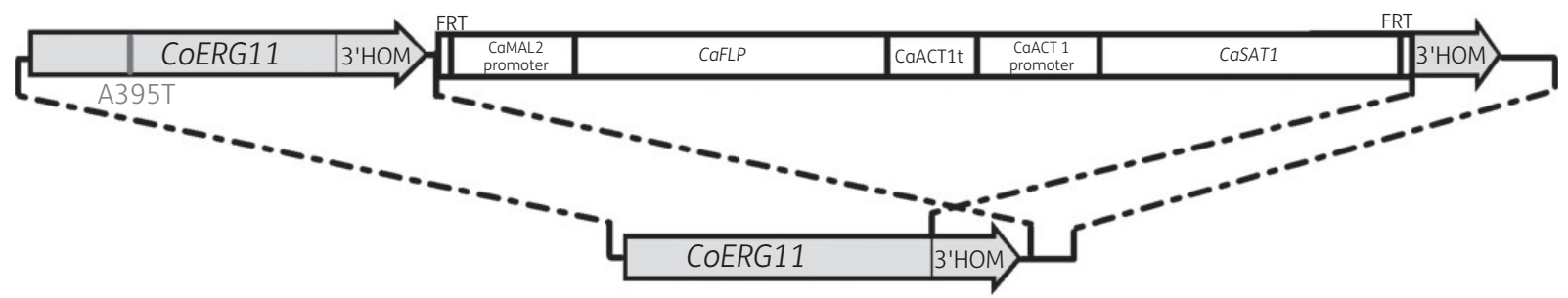

(b)

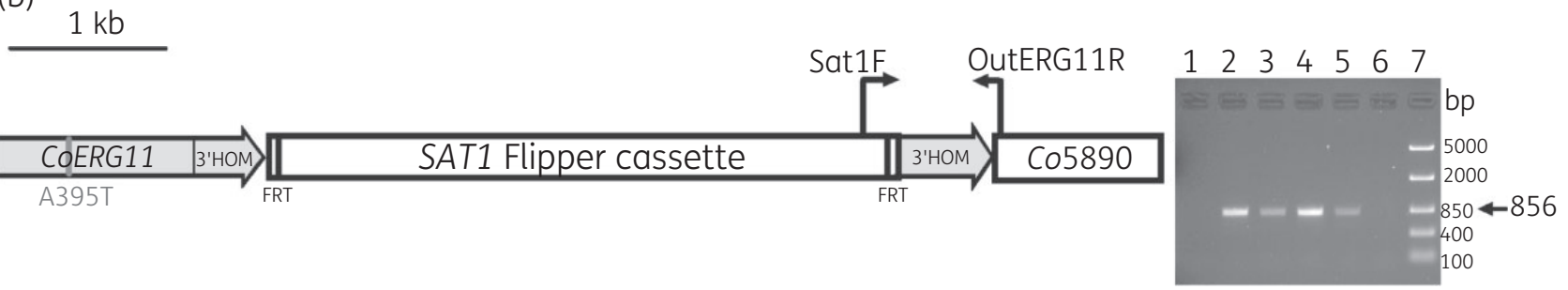

(c)

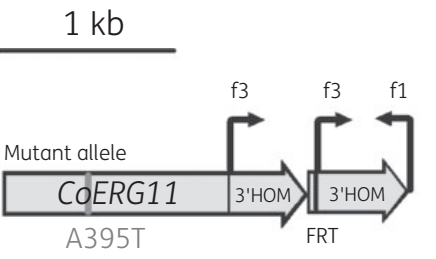

(d)

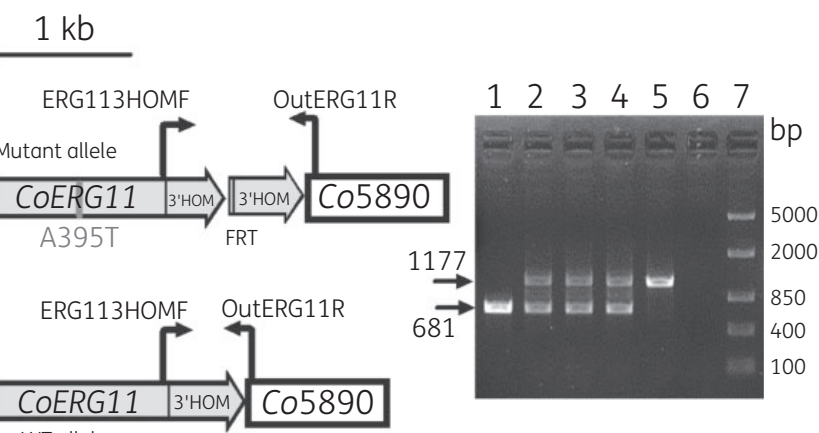

(e)

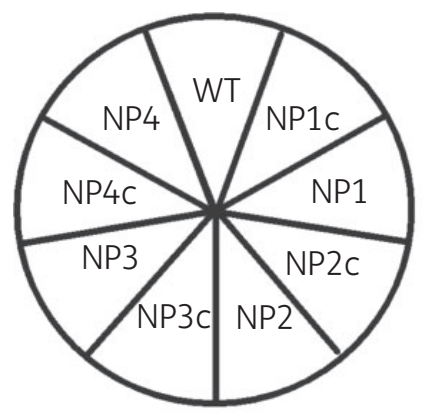

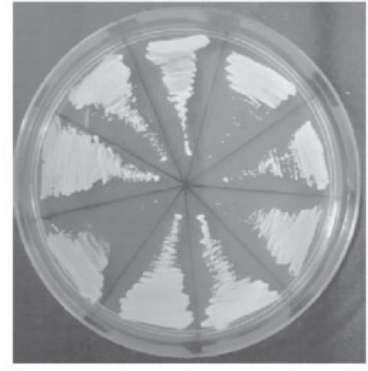

YPD

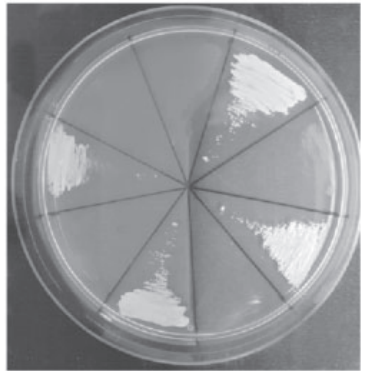

YPD+NTC

Figure 2. (a) Structure of the SAT1 flipper cassette used for the integration of CoERG11 gene carrying the A395T mutation. The cassette relies on a C. albicans adapted nourseothricin dominant resistant marker (CaSAT1) under the control of a C. albicans actin promoter (CaACT1). Recombinase FLP gene, whose expression is driven from the C. albicans MAL2 promoter gene (CaMAL2), results in the cassette excision by using flipper recombinase target sequences (FRT). Flanking downstream $3^{\prime}$ homology region contains the $3^{\prime}$ end of the CoERG11 gene and 217 bp flanking region outside the gene, while upstream $5^{\prime}$ region containing an entire copy of the CoERG11 containing the A395T mutation. (b) Evaluation of correct integration of SAT1 flipper cassette in CoERG11 locus by PCR, using primers Sat1F and OutERG11R. 1, Co287 WT; 2, Co287 NP1; 3, Co287 NP2; 4, Co287 NP3; 5, Co287 NP4; 6, negative control; 7, FastRuler Middle Range DNA Ladder (Thermo Scientific). The expected fragment size in mutant strains was 856 bp. (c) PCR attesting correct excision of SAT1 flipper cassette. 1, TrackIt ${ }^{\mathrm{TM}} 1 \mathrm{~kb}$ Plus DNA Ladder (Invitrogen); 2, Co287 WT; 3, Co287 NP1; 4, Co287 NP2; 5, Co287 NP3; 6, Co287 NP4; 7, negative control. Expected fragment sizes: $1070 \mathrm{bp}$ (mutated allele) and $574 \mathrm{bp}$ (WT allele). (d) Discrimination of heterozygous and homozygous strains carrying the A395T mutation. 1, Co287 WT; 2, Co287 NP1; 3, Co287 NP3; 4, Co287 NP4; 5, Co287 NP2; 6, negative control; 7, FastRuler Middle Range DNA Ladder (Thermo Scientific). Expected fragment sizes in heterozygous strains: 1177 and $681 \mathrm{bp}$. Expected fragment sizes in homozygous mutant strains: $1177 \mathrm{bp}$ and WT strain 681 bp. (e) WT and mutant strains grown on YPD plates and nourseothricin-supplemented YPD plates at $30^{\circ} \mathrm{C}$ for $24 \mathrm{~h}$. NP1c, NP2c, NP3c and NP4c, mutant strains containing the cassette; NP1, NP2, NP3 and NP4, mutant strains following flippase activation and cassette excision. NTC, nourseothricin. 
the SAT1 flipper cassette strategy (Figure 2a). Following the first round of transformation, four C. orthopsilosis Co287-derived clones (NP1-4) integrated the cassette into their genome since they were able to grow under selective conditions (nourseothricin/YPD plates). The correct cassette integration in the CoERG11 locus was verified by PCR (Figure 2b) performed on genomic DNA obtained from the NP1-4 clones. Following flippase activation, genomic DNA was extracted and cassette excision was verified by PCR (Figure 2c) and by the absence of growth onto nourseothricinsupplemented YPD plates (Figure 2e).

To verify the presence of the A395T mutation in the CoERG11 locus, the corresponding region was sequenced in all the clones obtained (Table 2). Three clones (NP1-3) carried the A395T mutation: the NP1 and NP3 clones were heterozygous for the A395T mutation (accession numbers MG543290 and MG543292, respectively), while clone NP2 was homozygous for the A395T mutation (accession number MG543291). PCR analysis further confirmed the replacement of both alleles with the mutated version of the gene in the NP2 clone (Figure 2d). Clone NP4, although correctly integrating the cassette in the CoERG11 locus, did not carry the mutation (Table 2, accession number MG543293).

\section{Phenotypic characterization of the NP1-4 mutant panel}

Antimicrobial susceptibility assay was performed for the panel of the obtained mutant clones (NP1-4) as well as for Co287 WT strain. A resistant phenotype to fluconazole (from 8 - to 16 -fold increase), itraconazole and voriconazole, was observed in clones NP1-3, while WT strain and clone NP4 did not show any change in susceptibility (Table 2). Interestingly, clone NP2, in which both alleles carried the A395T substitution, showed the highest MIC values of fluconazole (16-fold increase) and voriconazole ( $>30$-fold) among the mutant strains (Table 2). To verify that the increased resistance of mutants did not relate to a direct effect of the mutation on the basal growing ability, the growth of mutants NP1-3 was compared with that of Co287 WT isolate in YPD broth medium at $37^{\circ} \mathrm{C}$. No difference was observed in growth rate among the mutants and the WT strains (Figure 3).

\section{Discussion}

In the present study, a panel of $C$. orthopsilosis clinical isolates collected from two different geographical regions of Italy was selected and evaluated for azole antifungal susceptibility. A high number of multi-azole-resistant isolates was observed among isolates collected from both regions. This is interesting in view of recent reports indicating that resistant non-albicans Candida species are increasingly isolated in the hospital setting, most likely due to the selection of acquired resistance during medical treatments. It has been reported that $20 \%-30 \%$ of candidaemia cases involve species with intrinsic resistance to either fluconazole or echinocandins. ${ }^{36}$ To better understand the molecular mechanisms that underlie the azoleresistant phenotype in C. orthopsilosis, we sequenced the orthologue of C. albicans ERG11 (CORT OE05900) in both azole-resistant and azole-susceptible C. orthopsilosis clinical isolates. Indeed, mutations in the ERG11 sequence leading to amino acid substitutions in the encoded lanosterol $14 \alpha$-demethylase have been demonstrated to contribute to $C$. albicans azole resistance. ${ }^{12,37-40}$ Point mutations in ERG11 orthologues have also been associated with azole resistance

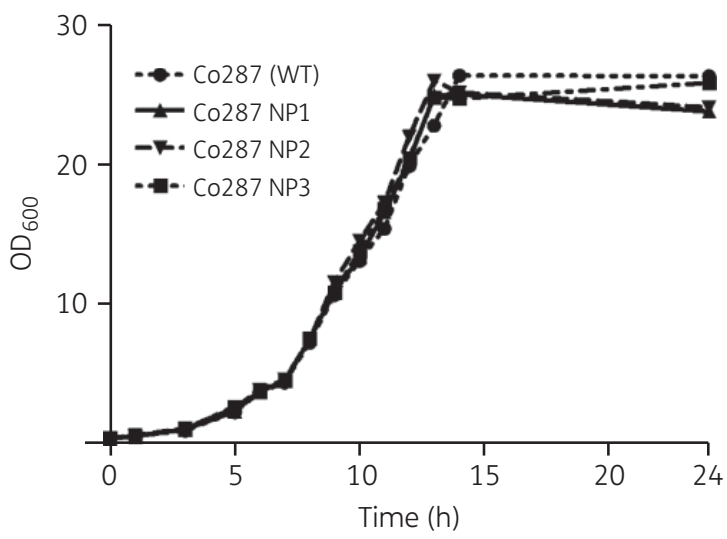

Figure 3. Growth curve of Co287 WT strain and mutant derivative clones Co287 NP1, NP2 and NP3 in YPD liquid medium at $37^{\circ} \mathrm{C}$.

in C. tropicalis, ${ }^{41}$ C. parapsilosis ${ }^{24}$ and, more recently, in the multiresistant Candida auris. ${ }^{42}$ However, no data are available on azole resistance in C. orthopsilosis. Sequencing results highlighted six mutations linked to non-synonymous amino acid substitutions in our panel of $C$. orthopsilosis clinical isolates, out of which only Y132F was predicted to be damaging and deleterious. This finding is in agreement with what has been previously described for other Candida species. ${ }^{12,24,37-39,41,42}$ Notably, the mutation was carried by azole-resistant isolates only (fluconazole MIC ranging between 8 and $128 \mathrm{mg} / \mathrm{L}$ ) and was never observed in azole-susceptible strains. Substitution of tyrosine with phenylalanine could lead to an increase in the hydrophobicity of the active site in Erg11p, resulting in a reduced affinity of the enzyme for fluconazole, which is a hydrophilic molecule.

To demonstrate that $\mathrm{Y} 132 \mathrm{~F}$ is responsible for azole resistance in C. orthopsilosis we used a SAT1 flipper-driven transformation to replace a WT copy of COERG11 with a functional copy of the gene carrying the A395T variant in a fluconazole-susceptible clinical isolate. Upon cassette excision from C. orthopsilosis genome, both heterozygous and homozygous clones were obtained, carrying one of either copy of the mutated CoERG11 allele, respectively. Phenotypic tests were performed on the panel of COERG11T mutant strains to rule out any potential effect of the induced mutation on growth ability in conventional media. Susceptibility testing indicated that C. orthopsilosis mutant clones carrying the 395T variant (NP1-3) acquired a resistant phenotype to fluconazole, with the homozygous strain showing the higher increase in fluconazole MIC (from 2 to $32 \mathrm{mg} / \mathrm{L}$ ). Moreover, the acquisition of one mutated CoERG11 allele was sufficient to induce an azole-multiresistant phenotype, with MIC values for itraconazole and voriconazole increasing from 0.125 and $0.03 \mathrm{mg} / \mathrm{L}$ (classified as susceptible) to 0.25 and $0.25-1 \mathrm{mg} / \mathrm{L}$ (classified as resistant), respectively. As expected, the homozygous CoERG11-395T mutated strain displayed the highest MIC values for azoles. The introduction of a mutated allele produced a net change in fluconazole susceptibility, from a susceptible to a resistant phenotype, although the mutated strains did not reach the fluconazole MIC value observed for the 'donor strain': this could be explained by the fact that the mutated allele is introduced in a completely different genomic background. Other C. orthopsilosis clinical 
isolates showed an azole-resistant phenotype in the absence of the Y132F substitution. Azole resistance can originate from different molecular mechanisms, e.g. overexpression of the target, of efflux pumps, or other alterations in sterol biosynthesis, as demonstrated for other Candida species, including C. parapsilosis. ${ }^{43}$ Future studies need to be undertaken to unravel the mechanisms underlying azole resistance in these clinical isolates.

In conclusion, this study presents, to the best of our knowledge, the first assessment of the molecular mechanisms involved in fluconazole resistance in clinical isolates belonging to the emerging species C. orthopsilosis, demonstrating that a Y132F substitution in CoErg11p is sufficient to confer multi-azole resistance.

\section{Acknowledgements}

This study was presented in part at the Twenty-Seventh European Congress of Clinical Microbiology and Infectious Diseases, Vienna, Austria, 2017 (Abstract P1756).

We thank Dr Colin Gerard Egan for carefully reading the manuscript and providing helpful suggestions (prior to submission) and Professor Joachim Morschhäuser for providing us with the SAT1 flipper cassette.

\section{Funding}

Financial support was obtained from the University of Pisa (grant no. PRA_2017_18 $\mathrm{n}^{\circ} 18$ ) and from the Italian Ministero dell'Istruzione, dell'Università e della Ricerca (MIUR) (research grant no. RBFR100FLV).

\section{Transparency declarations}

None to declare.

\section{Supplementary data}

Tables S1-S4 and Figure S1 are available as Supplementary data at JAC Online.

\section{References}

1 Brown GD, Denning DW, Gow NA et al. Hidden killers: human fungal infections. Sci Transl Med 2012; 4: 165rv13.

2 Posch W, Heimdorfer D, Wilflingseder D et al. Invasive candidiasis: future directions in non-culture based diagnosis. Expert Rev Anti Infect Ther 2017; 15: 829-38.

3 Tavanti A, Davidson AD, Gow NA et al. Candida orthopsilosis and Candida metapsilosis spp. nov. to replace Candida parapsilosis groups II and III. J Clin Microbiol 2005; 43: 284-92.

4 Trofa D, Gacser A, Nosanchuk JD. Candida parapsilosis, an emerging fungal pathogen. Clin Microbiol Rev 2008; 21: 606-25.

5 Espinel-Ingroff A, Pfaller MA, Bustamante B et al. Multilaboratory study of epidemiological cutoff values for detection of resistance in eight Candida species to fluconazole, posaconazole, and voriconazole. Antimicrob Agents Chemother 2014; 58: 2006-12.

6 Caggiano G, Coretti C, Bartolomeo N et al. Candida bloodstream infections in Italy: changing epidemiology during 16 years of surveillance. Biomed Res Int 2015; 2015: 256580.
7 Guinea J, Zaragoza O, Escribano P et al. Molecular identification and antifungal susceptibility of yeast isolates causing fungemia collected in a population-based study in Spain in 2010 and 2011. Antimicrob Agents Chemother 2014; 58: 1529-37.

8 Nucci M, Queiroz-Telles F, Alvarado-Matute T et al. Epidemiology of candidemia in Latin America: a laboratory-based survey. PLoS One 2013; 8: e59373.

9 Oliveira VK, Paula CR, Colombo AL et al. Candidemia and death by Candida orthopsilosis and Candida metapsilosis in neonates and children. Pediatr Neonatol 2014; 55: 75-6.

10 Manzoni P, Mostert M, Castagnola E. Update on the management of Candida infections in preterm neonates. Arch Dis Child Fetal Neonatal Ed 2015; 100: F454-9.

11 Perlin DS, Shor E, Zhao Y. Update on antifungal drug resistance. Curr Clin Microbiol Rep 2015; 2: 84-95.

12 Perea S, Lopez-Ribot JL, Kirkpatrick WR et al. Prevalence of molecular mechanisms of resistance to azole antifungal agents in Candida albicans strains displaying high-level fluconazole resistance isolated from human immunodeficiency virus-infected patients. Antimicrob Agents Chemother 2001; 45: 2676-84.

13 White TC. The presence of an R467K amino acid substitution and loss of allelic variation correlate with an azole-resistant lanosterol $14 \alpha$ demethylase in Candida albicans. Antimicrob Agents Chemother 1997; 41: 1488-94.

14 Xu Y, Chen L, Li C. Susceptibility of clinical isolates of Candida species to fluconazole and detection of Candida albicans ERG11 mutations. J Antimicrob Chemother 2008; 61: 798-804.

15 Kelly SL, Lamb DC, Kelly DE. Y132H substitution in Candida albicans sterol $14 \alpha$-demethylase confers fluconazole resistance by preventing binding to haem. FEMS Microbiol Lett 1999; 180: 171-5.

16 Kelly SL, Lamb DC, Loeffler J et al. The G464S amino acid substitution in Candida albicans sterol $14 \alpha$-demethylase causes fluconazole resistance in the clinic through reduced affinity. Biochem Biophys Res Commun 1999; 262: 174-9.

17 Asai K, Tsuchimori N, Okonogi K et al. Formation of azole-resistant Candida albicans by mutation of sterol 14-demethylase P450. Antimicrob Agents Chemother 1999; 43: 1163-9.

18 Xiao L, Madison V, Chau AS et al. Three-dimensional models of wild-type and mutated forms of cytochrome P450 14 $\alpha$-sterol demethylases from Aspergillus fumigatus and Candida albicans provide insights into posaconazole binding. Antimicrob Agents Chemother 2004; 48: 568-74.

19 Monk BC, Tomasiak TM, Keniya MV et al. Architecture of a single membrane spanning cytochrome P450 suggests constraints that orient the catalytic domain relative to a bilayer. Proc Natl Acad Sci USA 2014; 111: 3865-70.

20 Canton E, Peman J, Quindos G et al. Prospective multicenter study of the epidemiology, molecular identification, and antifungal susceptibility of Candida parapsilosis, Candida orthopsilosis, and Candida metapsilosis isolated from patients with candidemia. Antimicrob Agents Chemother 2011; 55: 5590-6.

21 Garcia-Effron G, Katiyar SK, Park S et al. A naturally occurring proline-toalanine amino acid change in Fks1p in Candida parapsilosis, Candida orthopsilosis, and Candida metapsilosis accounts for reduced echinocandin susceptibility. Antimicrob Agents Chemother 2008; 52: 2305-12.

22 Grossman NT, Pham CD, Cleveland AA et al. Molecular mechanisms of fluconazole resistance in Candida parapsilosis isolates from a U.S. surveillance system. Antimicrob Agents Chemother 2015; 59: 1030-7.

23 Silva AP, Miranda IM, Guida A et al. Transcriptional profiling of azoleresistant Candida parapsilosis strains. Antimicrob Agents Chemother 2011; 55: 3546-56.

24 Souza AC, Fuchs BB, Pinhati HM et al. Candida parapsilosis resistance to fluconazole: molecular mechanisms and in vivo impact in infected Galleria mellonella larvae. Antimicrob Agents Chemother 2015; 59: 6581-7. 
25 Branco J, Silva AP, Silva RM et al. Fluconazole and voriconazole resistance in Candida parapsilosis is conferred by gain-of-function mutations in MRR1 transcription factor gene. Antimicrob Agents Chemother 2015; 59: 6629-33.

26 Zhang L, Xiao M, Watts MR et al. Development of fluconazole resistance in a series of Candida parapsilosis isolates from a persistent candidemia patient with prolonged antifungal therapy. BMC Infect Dis 2015; 15: 340.

27 Subcommittee on Antifungal Susceptibility Testing of the ESCMID EUCAST. EUCAST definitive document EDef 7.1: method for the determination of broth dilution MICs of antifungal agents for fermentative yeasts. Clin Microbiol Infect 2008; 14: 398-405.

28 Tavanti A, Hensgens LA, Ghelardi E et al. Genotyping of Candida orthopsilosis clinical isolates by amplification fragment length polymorphism reveals genetic diversity among independent isolates and strain maintenance within patients. J Clin Microbiol 2007; 45: 1455-62.

29 Riccombeni A, Vidanes G, Proux WE et al. Sequence and analysis of the genome of the pathogenic yeast Candida orthopsilosis. PLoS One 2012; 7: e35750.

30 Kumar P, Henikoff S, Ng PC. Predicting the effects of coding nonsynonymous variants on protein function using the SIFT algorithm. Nat Protoc 2009; 4: 1073-81.

31 Kelley LA, Mezulis S, Yates CM et al. The Phyre2 web portal for protein modeling, prediction and analysis. Nat Protoc 2015; 10: 845-58.

32 Choi Y, Chan AP. PROVEAN web server: a tool to predict the functional effect of amino acid substitutions and indels. Bioinformatics 2015; 31: 2745-7.

33 Reuss O, Vik A, Kolter R et al. The SAT1 flipper, an optimized tool for gene disruption in Candida albicans. Gene 2004; 341: 119-27.

34 Bertini A, Zoppo M, Lombardi L et al. Targeted gene disruption in Candida parapsilosis demonstrates a role for CPAR2_404800 in adhesion to a biotic surface and in a murine model of ascending urinary tract infection. Virulence 2016; 7: 85-97.
35 Looke M, Kristjuhan K, Kristjuhan A. Extraction of genomic DNA from yeasts for PCR-based applications. Biotechniques 2011; 50: 325-8.

36 Arendrup MC. Update on antifungal resistance in Aspergillus and Candida. Clin Microbiol Infect 2014; 20 Suppl 6: 42-8.

37 Chau AS, Mendrick CA, Sabatelli FJ et al. Application of real-time quantitative PCR to molecular analysis of Candida albicans strains exhibiting reduced susceptibility to azoles. Antimicrob Agents Chemother 2004; 48: 2124-31.

38 Flowers SA, Colon B, Whaley SG et al. Contribution of clinically derived mutations in ERG11 to azole resistance in Candida albicans. Antimicrob Agents Chemother 2015; 59: 450-60.

39 Goldman GH, da Silva Ferreira ME, dos Reis Marques E et al. Evaluation of fluconazole resistance mechanisms in Candida albicans clinical isolates from HIV-infected patients in Brazil. Diagn Microbiol Infect Dis 2004; 50: 25-32.

40 Sanglard D, Ischer F, Koymans $L$ et al. Amino acid substitutions in the cytochrome P-450 lanosterol 14 $\alpha$-demethylase (CYP51A1) from azoleresistant Candida albicans clinical isolates contribute to resistance to azole antifungal agents. Antimicrob Agents Chemother 1998; 42: 241-53.

41 Tan J, Zhang J, Chen W et al. The A395T mutation in ERG11 gene confers fluconazole resistance in Candida tropicalis causing candidemia. Mycopathologia 2015; 179: 213-8.

42 Lockhart SR, Etienne KA, Vallabhaneni S et al. Simultaneous emergence of multidrug-resistant Candida auris on 3 continents confirmed by wholegenome sequencing and epidemiological analyses. Clin Infect Dis 2017; 64: 134-40.

43 Berkow EL, Manigaba K, Parker JE et al. Multidrug transporters and alterations in sterol biosynthesis contribute to azole antifungal resistance in Candida parapsilosis. Antimicrob Agents Chemother 2015; 59: 5942-50. 